\title{
A microcomputer-based plotter system for the TRS-80 and HP 7225
}

\author{
ROBERT L. MAPOU and LARRY D. BYRD \\ Yerkes Regional Primate Research Center, Emory University, Atlanta, Georgia 30322
}

\begin{abstract}
A software package, MICROPLO'T, was developed to run on a TRS-80 Model I microcomputer in conjunction with the Hewlett-Packard (HP) 7225 or similiar HP plotter. Capabilities include XY plotting of up to 10 data sets and curve plotting of an nth-degree polynomial. The package operates under the NEWDOS 80 Version 2.0 disk operating system but, with appropriate modification, can be run under any operating system that has dynamic program line merge and delete functions in BASIC. This report describes the structure of the system, hardware configuration, plotting capabilities, and applications in the behavioral laboratory.
\end{abstract}

The application of microcomputers in the behavioral laboratory and the availability of inexpensive plotters have made feasible automatic data plotting with minimal investment. Examples of hardware/software configurations have been described by Hayes (1981) and Palya and Brown (1981) for DEC microcomputers, and Durrett (1980) has presented information on an inexpensive plotter.

One microcomputer system presently used in many behavioral laboratories is the Radio Shack TRS-80 (Model I), a Z-80-based microcomputer with many sophisticated features, including disk capability and RS-232-C interface compatibility. The RS-232-C allows the TRS- 80 to be connected to and communicate with a range of peripheral devices, including plotters.

The Hewlett-Packard (HP) 7225 is a versatile, yet reasonably priced, graphics plotter ${ }^{1}$ that is provided to the user with a set of plotter instructions. The user must provide a means of communication between the plotter and computer and write programs that send the appropriate instructions to the plotter. Attractive features of the plotter include the ability to generate lettering from one of five character sets without additional user programming, versatility in scaling plot size to user requirements, and the ability to digitize data, that is, to determine the coordinates of points from user-provided plots (see Durrett, 1980) and transmit the coordinates back to the computer.

Three steps can be identified in the process of developing a microcomputer-based plotting system. First, the hardware requirements must be specified. Second, software must be developed to permit communication between the computer and plotter. Third,

Development of this sytem was supported by U.S. Public Health Service Grants DA 01161, DA 02128, and RR 00165 (Division of Research Resources, National Institutes of Health). Thanks to M. D. Zeiler, T. F. Blakely, and J. B. Richards for comments during the preparation of this paper. Reprints may be obtained from Larry D. Byrd, Yerkes Regional Primate Research Center, Emory University, Atlanta, Georgia 30322. a set of software routines must be developed that generate commands in the plotter's instruction set in the order necessary to do the user-requested plot. The MICROPLOT package described here for use with the TRS-80 and HP 7225 plotter can be characterized according to these three processes.

\section{HARDWARE AND OPERATING SYSTEM REQUIREMENTS}

MICROPLOT requires a 48-KB TRS-80 Model I microcomputer and expansion interface with a RS-232$\mathrm{C}$ card installed. ${ }^{2}$ A minimum of two disk drives is required, with Drive 0 reserved for the operating system and plotter software and Drive 1 and above used for data files. The HP 7225 plotter must include the $17603 \mathrm{~A}$ personality module. It is assumed that the user has access to all equipment manuals to insure clarification of the hardware configuration and connections.

MICROPLOT operates under NEWDOS 80 Version 2.0 and makes use of the feature of NEWDOS 80 that allows dynamic merging and deletion of program lines in BASIC. This feature decreases the amount of memory occupied by the program at any one time because sections of the software can be swapped in and out of memory as needed. With appropriate modification, MICROPLOT can be run under any disk operating system that includes dynamic delete and merge functions in BASIC.

The HP 7225 plotter contains a 630-byte buffer that can receive data from the TRS- 80 . Because the rate of data transmission from the computer is much faster than the rate of plotting, that is, the rate at which the buffer is emptied, some method of "handshaking" must be employed that allows the plotter to communicate the status of its buffer to the computer. This status can then be used by the computer to determine whether data transmission should continue or whether the computer should wait until additional buffer space is available. Software for "handshaking" is included in MICROPLOT and is invisible to the user. Transmission 
Table 1

Function and Visibility to the User of Routines Comprising the MICROPLOT System

\begin{tabular}{|c|c|}
\hline Name & Function \\
\hline & Routines That are Visible to the User \\
\hline MAIN & Overall controller providing menu presentation, merging, and calling of subroutines \\
\hline AXISINIT & Initializes and alters axis parameters from keyboard \\
\hline AXISREAD & Initializes axis parameters from file \\
\hline DSINPUT & Inputs data for plotting from keyboard or file \\
\hline XYPLOT & Calls all routines necessary to plot one or more data sets \\
\hline CURVPLOT & Calls all routines necessary to plot an nth-degree polynomial \\
\hline \multirow[t]{2}{*}{ PLOTINIT } & Loads PLOTBOOT and sets default parameters \\
\hline & Routines That are Invisible to the User \\
\hline AXIS & Plots and labels an $\mathrm{X}$ or $\mathrm{Y}$ axis \\
\hline DATAPLOT & Plots one or more data sets \\
\hline VARPLOT & Plots a variability bar at a data point \\
\hline TITLE & Prints a plot title \\
\hline PLOTU & Moves pen to an absolute or relative plotter coord inate with pen up or down \\
\hline PLOTBOOT & Assembly language plotter communication routine \\
\hline
\end{tabular}

a

Example of XY FIot with Variability flare - Four Quadrant.

Line 1. $y=x$ (Data pointt o)

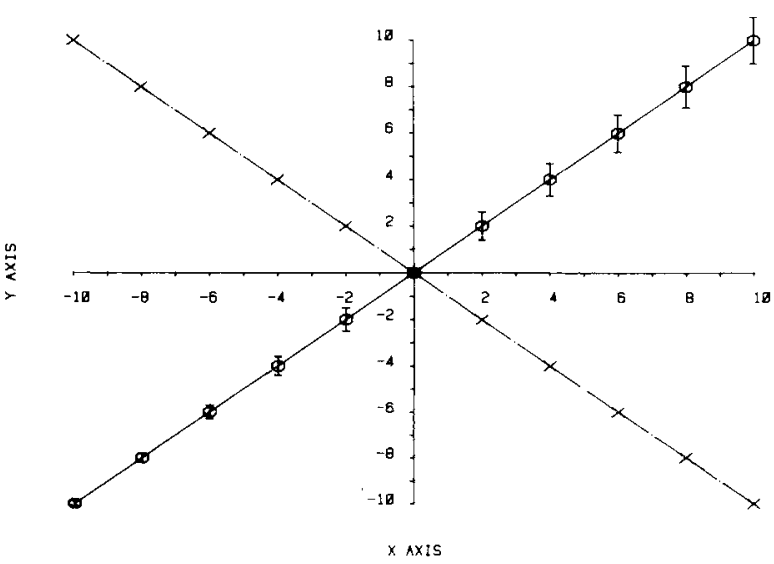

FR 30 TO 40 S S 250
SESSION NOTES, SALINE I. M.

$c$

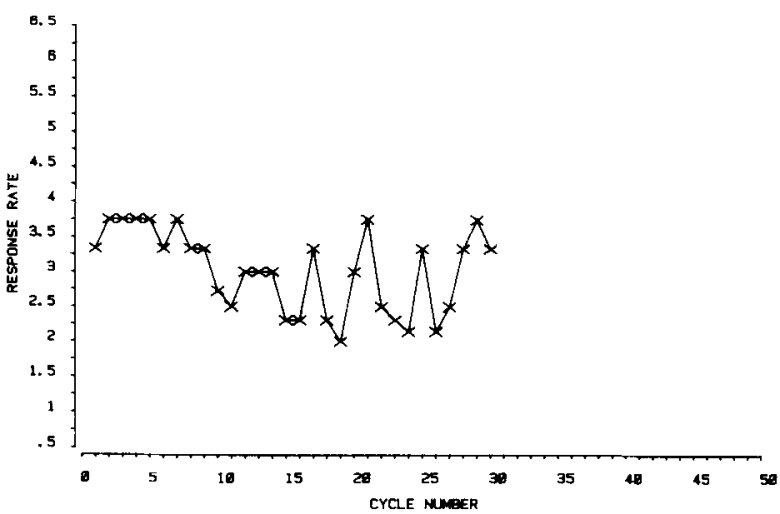

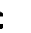

.

Example of Eurve Plot of $Y=X \times X$ Over Plotted Doto Pointe Curve is compoesed of 58 pointe.

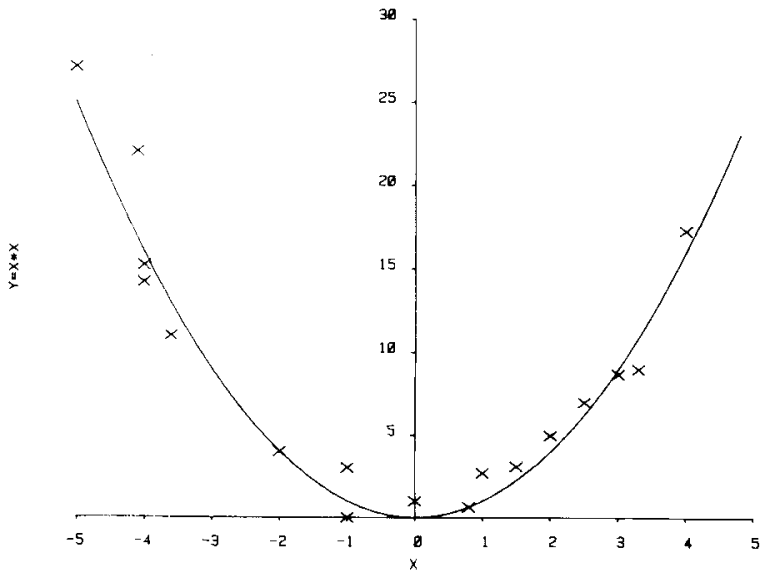


of plotter commands takes place through an assembly language subroutine that is loaded into a reserved area of high memory. The routine is accessed through the LPRINT command in the plotter software.

With the exception of the "handshaking" routine, the plotter software is written in NEWDOS 80 Version 2.0 BASIC. The software consists of a main program and a group of subroutines, each of which has a given function (e.g., to input data, to input plot characteristics, to draw an axis, to plot a data set). The main program merges these subroutines into memory for a given task and calls them in the required order. At any time, the only subroutines occupying memory are those required for a given task. In this manner, required memory space is kept at a minimum. Table 1 describes the system routines.

\section{SYSTEM CAPABILITIES}

System communication with the user is interactive and self-explanatory. A menu is displayed on the video monitor following initialization. Task alternatives presented on the menu include initialization of axis parameters from the keyboard or a file, data set input from the keyboard or a file, straight-line XY plotting of one or more data sets, and plotting of an nth-degree polynomial (up to the ninth degree) using coefficients provided by the user. The latter two tasks prompt the user for all data necessary for completion of a plot, including loading the assembly language "handshaking" subroutine, axis parameter initialization, and data set input. The menu is displayed again following completion of a task.

The user may vary any of the following characteristics of a plot: minimum and maximum axis values, axis length, point of axes intersection, spacing between axes at the intersection point, distance between ticks (slash marks on an axis), distance between tick labels, axis labels (automatically centered), plot title (up to 10 lines), type of line used to connect data points (seven are available, including no line), type of character plotted at each point (none, $o, x,+, *$, \#), and whether variability is to be shown for each data point. Character size and letter size are scaled automatically and are always proportional to the size of the plot. Several sample plots are shown in Figure 1.

One application of the system in this laboratory is the plotting of data derived from schedule-controlled performance. Conditioned responding is controlled on-line by a TRS-80, and data are stored in a standard format on disk. The plotting task includes input of a standard set of parameters used to draw axes and to label each plot, input of data from the disk data file for the session, computation of response rates, and plotting. A plot produced by this task appears in Figure 1c. Because of the modular design of the software, similar tasks can be designed easily to accommodate any customized application.

Detailed information concerning utilization and availability of the package is available from the authors.

\section{REFERENCES}

DuRRETT, H. J. Inexpensive plotters and digitizers for psychological research and instruction. Behavior Research Methods \& Instrumentation, 1980, 12, 244-247.

HAYEs, W. GRAPH: A BASIC-11 graphics program for digital plotters. Behavior Research Methods \& Instrumentation, 1981, 13, 367.

Palya, W. L., \& Brown, B. Graphics software and hardware for RT-11 systems. Behavior Research Methods \& Instrumentation, 1981, 13, 255-261.

\section{NOTES}

1. Detailed specifications of plotter hardware, software, and external hardware and software requirements are available from local HP offices or from Hewlett-Packard, 16399 W. Bernardo Drive, San Diego, California 92127. List price as of April 1982 was $\$ 3,500$.

2. It is assumed that MICROPLOT will also run on the TRS-80 Model III with minimal modification.

(Received for publication March 16, 1982; revision accepted May 7, 1982.) 